\title{
Review: parent training and community-based interventions may benefit children with disruptive behaviour disorders
}

Farmer EM, Compton SN, Burns BJ, Robertson E. Review of the evidence base for treatment of childhood psychopathology: externalizing disorders.J Consult Clin Psychol. 2002 Dec; 70: 1267-302.

\section{QUESTION: Which interventions are effective for children aged 6-12 years with externalising behavioural disorders?}

\section{Design}

Systematic review with narrative synthesis.

\section{Data sources}

The authors searched Medline, PsycInfo and the reference lists of identified articles. Expert reviewers suggested additional studies.

\section{Study selection}

Controlled trials of non-residential treatments for children with externalising behavioural disorders were eligible if they were published in English language journals between 1985-2000; included participants with mean age between 6 and 12 years; focused on community-based or outpatient settings, and reported on specific interventions for youths, their families or both. The authors addressed two main subsets of externalising orders: disruptive behaviour disorders (conduct disorder, oppositional defiant disorder) and attention deficit hyperactivity disorder (ADHD). Participants with comorbid conditions were categorised under the primary defining condition. Exclusion criteria were less than 30 participants (except in psychosocial or adjunctive treatments for ADHD); majority of sample outside the 6-12 year range, and insufficient outcome data.

\section{Data extraction}

The authors did not describe how data were extracted or how many reviewers performed the data extraction.

\section{Main results}

The authors identified 28 studies focused on interventions for children with ADHD and 21 interventions for disruptive behavioural disorders. Findings suggest that parent training and community-based interventions may be beneficial for disruptive behaviour disorders. Medication may be most effective for children with ADHD.

\section{Conclusions}

There is evidence that a variety of interventions are effective for children with externalising behavioural disorders. The authors suggest a need for further research on the effectiveness of specific interventions and strategies to enhance implementation.

\section{COMMENTARY}

These two reviews examine whether treating internalising and externalising disorders in $6-12$ year old children decreases the likelihood of substance abuse in adolescence and young adulthood. The authors investigated both psychosocial and psychopharmacological treatments. They provide detailed tables listing the characteristics of individual studies. The overall conclusion is that there are insufficient data to establish a preventative link between treating childhood disorders and substance abuse in adolescence.

The reviews underscore an important and yet to be exploited agenda, namely, evaluating the broader and long term impact of treating children effectively. Substance abuse and a variety of other social, emotional, behavioural, and medical problems might be influenced by treating childhood disorders effectively.

The paucity of long term follow up is a cogent complaint in child, adolescent, and adult therapy literature. There are many obstacles, including retaining a large and not too biased cohort, obtaining funding for long term follow up, and maintaining a control or standard treatment group as a comparison. These reviews highlight the importance of follow up in light of the possible, but yet to be investigated, implications for preventing other disorders and presumably reducing service use. The reviews are likely to prompt research to address this topic.

A key issue is whether and how to move from understanding risk factors to planning intervention strategies. Internalising and externalising disorders among children are precursors of, and comorbid with, substance abuse. This could mean that intervening in the disorders would impact on substance abuse, but it could also mean that it would not. Identifying risk factors and correlates is an interesting and useful point of departure, but many risk factors have no causal role in the onset or maintenance of a problem. The reviews sensitise us to the implications of child treatment, the urgent need to assess broader outcomes beyond the focus of the intervention, and the important role that treatment may play in prevention.

Professor Alan E Kazdin, PhD Director, Child Study Center Yale University School of Medicine Connecticut, USA
Source of funding: National Institute on Drug Abuse, Bethesda, Maryland, USA.

For correspondence: E Farmer, Services Effectiveness Research Program, Department of Psychiatry and Behavioral Sciences, Duke University School of Medicine, Durham, North Carolina, USA bfarmer@psych.mc.duke. edu 